\title{
The use of a porous medium method for modeling fixed-bed reactors with a low tube-to-particle diameter ratio
}

\author{
$\underline{T y m o e u s z ~ S ́ w i e b o d a ~}{ }^{1}$, Renata Krzyżyńska ${ }^{1}$, Anna Bryszewska-Mazurek ${ }^{1}$, \\ Wojciech Mazurek ${ }^{1}$
}

1. Faculty of Environmental Engineering, Wroclaw University of Science and Technology, POLAND,

Wrocław, 27 Wybrzeze Wyspianskiego St., E-mail: tymoteusz.swieboda@pwr.edu.pl

\begin{abstract}
The paper presents a method of modeling fixed-bed reactors based on the porous medium with an anisotropic thermal conductivity coefficient. The calculation results of the pressure drop and heat transfer of the simplified models were compared to the reference models results. As a comparative criterion, it was proposed to use area-weighted average pressure and mass-weighted average temperature.
\end{abstract}

Keywords - fixed-bed reactor, porous media, anisotropic thermal conductivity coefficient.

\section{Introduction}

Fixed-bed reactors are used in a wide range of technological processes, such as separation, purification, and chemical reactors. Among them, a special place is occupied by the devices with a low tube-to-particle diameter ratio $(N<10)$. While creating a computational mesh based on geometries special attention should be paid to the areas in the vicinity of the contact points of the bed elements $[1,2]$.

The porous medium seems to be an appropriate tool for modeling the fixed-bed reactors in engineering applications. This work presents a method to compensate for the impact of wall effects and local back-flows, which, at devices with small values of the tube-to-particle-diameter ratio $(N)$, severely affect the heat flow.

\section{Methods}

The starting point for the described procedure was the reactor described in [3], in the shape of a cylinder in $20 \mathrm{~mm}$ in diameter filled with glass spheres $\left(\lambda=1.1 \mathrm{~W} \cdot \mathrm{K}^{-1} \cdot \mathrm{m}^{-1}\right)$. The outer walls of the reactor were heated up to $400^{\circ} \mathrm{C}$ and its inlet was fed from the top with air at $60^{\circ} \mathrm{C}$.

a)

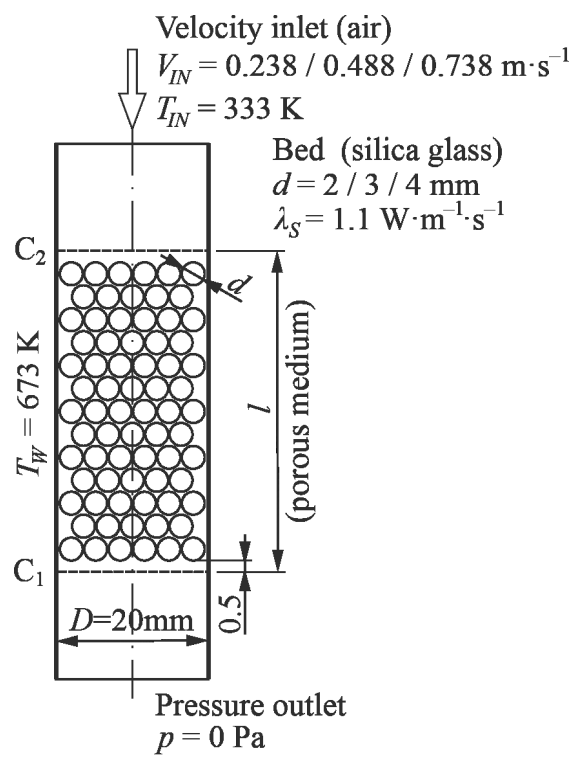

b)

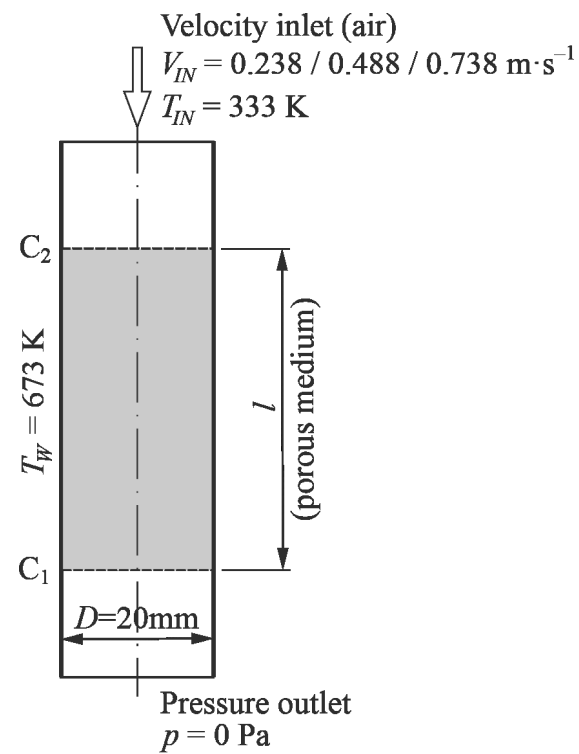

Fig. 1. Schematic of modeled fixed-bed reactor: a) reference model, b) simplified model 
Two sets of geometries were prepared for simulations. The first one consisted of nine variants of detailed bed geometries to use as reference models. The variants differed in the diameters of the filling spheres $(\mathrm{d}=2,3$ and $4 \mathrm{~mm})$ and the number of layers of the spheres $(5,9$ and 13) details in Fig. 1a. The second set contained nine variants of simplified geometries in which zones were dedicated to the porous medium. The length of each zone corresponded to the bed height in the reference model (see Fig 1b).

The area-weighted average (AWA) pressure and mass-weighted average (MWA) temperature were defined to enable the comparison of results from both model sets in planes $\mathrm{C}_{1}$ and $\mathrm{C}_{2}$.

$$
\begin{aligned}
& A W A(\phi)=\frac{1}{A} \int \phi d A=\frac{1}{A} \sum_{i=1}^{n} \phi_{i}\left|A_{i}\right| \\
& M W A(\phi)=\frac{\int \phi \varrho|\vec{v} \cdot d \vec{A}|}{\int \varrho|\vec{v} \cdot d \vec{A}|}=\frac{\sum_{i=1}^{n} \phi_{i} \varrho_{i}\left|\vec{v}_{i} \cdot \vec{A}_{i}\right|}{\sum_{i=1}^{n} \varrho_{i}\left|\vec{v}_{i} \cdot \vec{A}_{i}\right|}
\end{aligned}
$$

\section{Results and discussion}

In the first stage, a series of simulations were performed to obtain the distribution of pressure and temperature within the reference model of the bed. For each geometry variant, three simulations were carried out at different inlet air velocities $(0.238,0.488$, and $0.738 \mathrm{~m} / \mathrm{s})$. Obtained pressure drops and air temperature increases calculated between cross-sections $\mathrm{C}_{1}$ and $\mathrm{C}_{2}$ were later used to determine the parameters of the porous medium (internal resistance, viscous resistance).

In the second stage, a series of analogous simulations were performed using simplified models with a standard porous medium. Thus, the second set of AWA pressure drop values and MWA temperature increase values was obtained. Comparing them with the values from reference models showed a satisfactory agreement of the obtained pressure drops (the discrepancies did not exceed $20 \%$ - in most cases below 13\%). It was different in the case of the air temperature increase values within the bed: simplified models gave results up to $200 \%$ higher than the corresponding reference models. Most likely these differences were caused by the inhomogeneous flow field within the bed including anomalies such as channelling, wall effects, local back-flows that occurred in the vicinity of the heated walls of the reactor.

a)

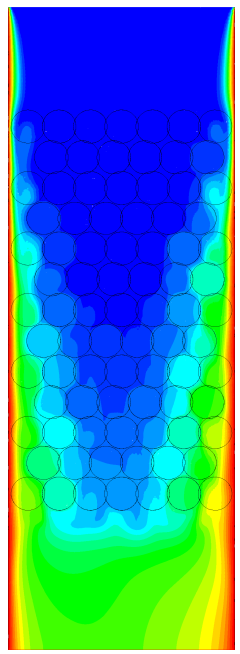

b)

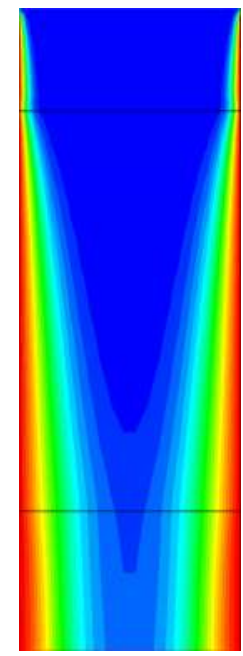

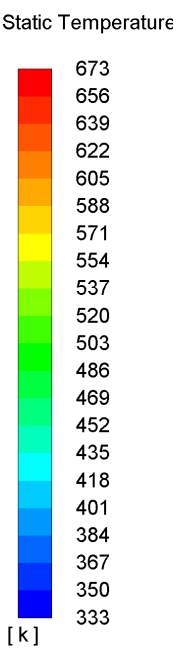

c)

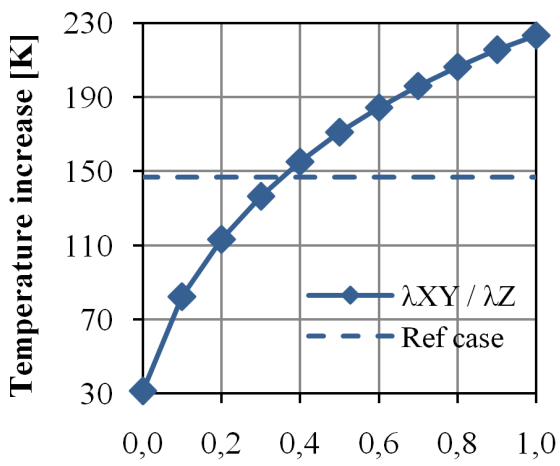

Relative thermal conductivity (in direction normal to flow)

Fig. 2. Comparison of exemplary temperature field simulation results: a) reference and b) simplified model temperature field, c) $\mathrm{C}_{1}-\mathrm{C}_{2}$ temperature increase error 
Taking into account the nature of the anomalies described, the simplified models incorrectly reproduced the heat exchange in the direction perpendicular to the general direction of the fluid flow. Therefore, it was proposed to introduce into the porous medium an anisotropic thermal conductivity coefficient. Reducing the value of its perpendicular components (relative values in range $0.12-0.28$ of the nominal value of the component in the flow direction - Fig. 2) made it possible in each case under consideration to obtain full compliance of the MWA temperature increase in the simplified model with the results of the reference model. The detailed results comparison is shown in Fig. 3a-c.

a)

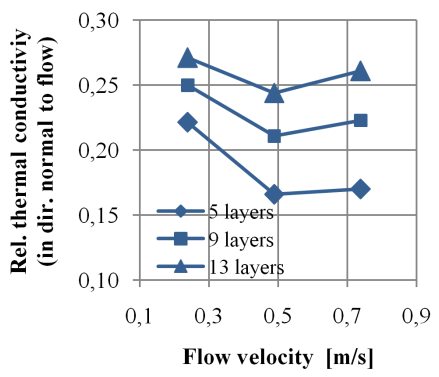

b)

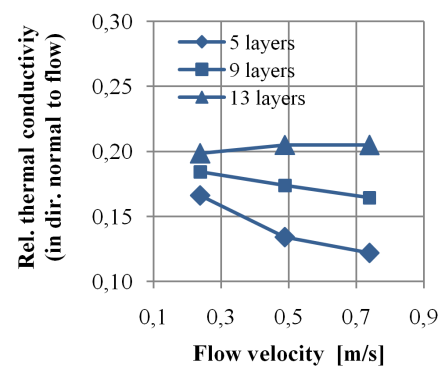

c)

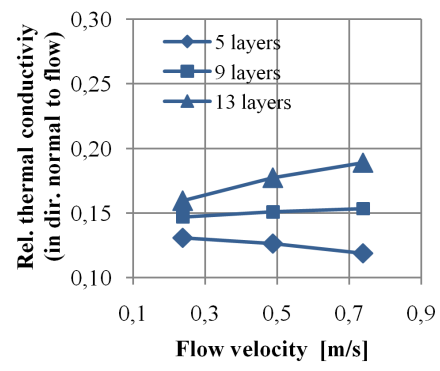

Fig. 3. Relative transverse thermal conductivity of porous medium as a function of air flow velocityfor a bed containing balls with a diameter of: a) $2 \mathrm{~mm}$, b) $3 \mathrm{~mm}$, c) $4 \mathrm{~mm}$

\section{Conclusion}

Models of fixed-bed reactors based on the standard porous medium method allow obtaining satisfactory consistency of results concerning pressure losses. Significant improvement of accuracy of determination of average outlet temperature can be obtained by using the proposed in this paper anisotropic thermal conductivity method. For model validation, the areaweighted average pressure and mass-weighted average temperature were chosen as comparison parameters.

\section{Acknowledgments}

This work was financed from project WND-POWR.03.02.00-00-I003/16-01 "InterDok Interdisciplinary Doctoral Studies Programs at the Wroclaw University of Science and Technology"

\section{References}

[1] Rebughini, S., Cuoci, A., Maestri, M. (2016). Handling contact points in reactive CFD simulations of heterogeneous catalytic fixed bed reactors. Chemical Engineering Science, 141, 240-249. https://doi.org/10.1016/j.ces.2015.11.013

[2] Dixon, A. G., Nijemeisland, M., Stitt, E. H. (2013). Systematic mesh development for 3d CFD simulation of fixed beds: Contact points study. Computers Chemical Engineering, 48, 135-153. https://doi.org/10.1016/j.compchemeng.2010.12.006

[3] Dong Y., Sosna B., Kroup O., Rosowski F., Horn R. (2017). Investigation of radial heat transfer in a fixed-bed reactor: CFD simulations and profile measurements. Chemical Engineering Journal, 317, 204-214. https://doi.org/10.1016/j.cej.2017.02.063 